\title{
Retos y aciertos de los fondos concursables para investigación: el caso de Costa Rica 2006-2016
}

\author{
Lizette Brenes Bonilla, Vicerrectoría de Investigación, UNED, 2050 San José, Costa Rica, \\ lbrenesb@gmail.com \\ Julián Monge-Nájera, Laboratorio de Ecología Urbana, Vicerrectoría de Investigación, UNED, \\ 2050 San José, Costa Rica; julianmonge@ uned.ac.cr; julianmonge@gmail.com
}

\begin{abstract}
In 2005 Costa Rican public universities decided that, instead of working in isolation, they would pool resources to finance joint research to solve environmental problems and support groups in disadvantage such as women and minorities. To examine the results after the first ten years of the program, we surveyed project coordinators and obtained replies from about half of them ( $\mathrm{N}=71$ projects). We found that $60 \%$ of research funds benefited disadvantaged populations (i.e. those in coastal and border areas) and that $54 \%$ of researchers who received funds were female. The 71 projects that completed the survey produced 1388 scientific articles and 883 practical products such as community workshops and commercial star-ups (estimate: over 2700 articles and 1700 practical products for the whole set of projects). These projects have been highly productive, have benefited women and underserved communities, led to lasting collaboration among researchers from different institutions, and are an example for other small countries with limited research funds.
\end{abstract}

Key words: fund distribution, research and development, women in science, science for underserved communities, academic cooperation in the tropics.

Resumen: En 2005, las universidades públicas costarricenses decidieron que, en lugar de trabajar aisladamente, reunirían recursos para financiar investigaciones conjuntas para resolver problemas ambientales y apoyar a grupos en desventaja, como mujeres y minorías. Para examinar los resultados de la primera década del programa, encuestamos a los coordinadores de proyectos y obtuvimos respuestas de aproximadamente la mitad de ellos ( $\mathrm{N}=71$ proyectos). Descubrimos que el $60 \%$ de los fondos de investigación beneficiaron a poblaciones desfavorecidas (es decir, las de las zonas costeras y fronterizas) y que el 54\% de los investigadores que recibieron fondos eran mujeres. Los 71 proyectos que completaron la encuesta produjeron 1388 artículos científicos y 883 productos prácticos como talleres comunitarios y arranques comerciales (estimación: más de 2700 artículos y 1700 productos prácticos en total). Estos proyectos han sido altamente productivos: han beneficiado a mujeres y comunidades marginadas; han llevado a una colaboración duradera entre investigadores de diferentes instituciones, y son un ejemplo para otros países pequeños con fondos de investigación limitados.

Palabras clave: distribución de fondos, investigación y desarrollo, mujeres en la ciencia, ciencia para comunidades marginadas, cooperación académica en los trópicos.

\section{Introducción}

La producción científica centroamericana se encuentra en crecimiento, aunque existen grandes diferencias entre países y un dominio visible de los hombres tanto por publicaciones como por nivel académico e impacto (Brenes-Bonilla, 2018). Por ejemplo, la productividad per cápita de 
Costa Rica o Panamá es diez veces mayor que la de cualquiera de sus vecinos centroamericanos, aunque en el caso de Panamá hay un sesgo por la presencia del Smithsonian Tropical Research Institute, un poderoso centro extranjero de investigación (Monge-Nájera \& Ho, 2012, 2016).

Las diferencias también se dan en cuanto a temas investigados: Guatemala, Honduras y Nicaragua hacen el énfasis en salud y agricultura típico de los países poco desarrollados (MongeNájera \& Ho, 2017a,b; Monge-Nájera \& Ho, 2018).

En todos los países centroamericanos hay una fuerte dependencia de los Estados Unidos y Europa, cuyos científicos dirigen la mayoría de los equipos de investigación de alta visibilidad, aunque el efecto es menos marcado en Costa Rica, que tiene el mayor desarrollo científico del istmo (Monge-Nájera \& Ho, 2012). También presentan condiciones desventajosas Guatemala, por las pocas revistas científicas propias (Monge-Nájera \& Ho, 2018), y Nicaragua, por la baja participación femenina en la ciencia y en la tecnología (Monge-Nájera \& Ho, 2017a). El Salvador es un caso atípico porque su producción principal se concentra en las ciencias sociales, por lo que posiblemente allí la presencia femenina sea mayor (Monge-Nájera \& Ho, 2017c).

Para aumentar la cooperación, la productividad y la participación femenina, las universidades públicas de Costa Rica han experimentado con un fondo especial concursable, conocido como Fondo del Sistema. Es parte del FEES, el Fondo Especial para la Educación Superior. Desde el 2005 promueve la integración de los talentos y recursos de las universidades públicas para enfrentar los retos nacionales. Para saber si el experimento ha tenido éxito, en este artículo analizamos quienes recibieron los recursos y cuáles beneficios produjeron en sus primeros 10 años de funcionamiento.

\section{MÉTODOS}

Las vicerrectorías de investigación de las cuatro universidades públicas participantes enviaron una encuesta digital a todos los coordinadores de proyecto beneficiados con fondos en esos diez años, indicando que su cooperación sería tomada en cuenta en futuras solicitudes. Analizamos los datos con Excel para general gráficos y buscar visualmente las tendencias a lo largo del tiempo. En el Apéndice Digital 1 se presentan en detalle los gráficos por total y los cocientes por proyectos.

\section{RESULTADOS}

Un obstáculo para obtener respuestas fue que, tras diez años, algunos coordinadores ya no estaban disponibles por cambio de empleo, retiro o fallecimiento. Sin embargo, obtuvimos una buena muestra porque el $45 \%$ de los 157 equipos beneficiados respondió la encuesta. Acá presentamos el detalle de quienes fueron beneficiados con los fondos (a nivel de investigadores y comunidades) y cuáles fueron los productos de esta, la mayor inversión en investigación de Costa Rica.

Personal beneficiado: A nivel de profesionales de investigación, la institución más beneficiada fue la Universidad de Costa Rica con 215 personas, seguida de la Universidad Nacional con 191, el Instituto Tecnológico de Costa Rica con 84 y finalmente la Universidad Estatal a Distancia con 57. Cabe destacar que la Universidad Estatal a Distancia no contaba con sistema de investigación durante los primeros cinco años de existencia de este fondo. 
En cuanto al género, predominaron las mujeres ( 572 mujeres beneficiadas, así: 285 Investigadoras y 287 estudiantes); los hombres estuvieron cerca de la paridad ( 485 hombres: 276 investigadores, 209 estudiantes). El $39 \%$ de las coordinaciones estuvieron a cargo de mujeres, pero la tendencia histórica es hacia la paridad en años recientes (Apéndice 1).

Comunidades beneficiadas: La mayoría de las comunidades beneficiadas están en las zonas con mayor necesidad de desarrollo, con un $48 \%$ (zonas costeras $28 \%$, zonas fronterizas $20 \%$ ). El $70 \%$ de los cantones con bajo desarrollo humano están en estas zonas y forman los conglomerados de mayor desigualdad (Brenes-Bonilla et al., 2015). La Gran Área Metropolitana solo recibió un $32 \%$ de los proyectos.

Productos: Los resultados transferidos a los grupos meta fueron principalmente reuniones con sectores y comunidades ( $\mathrm{N}=337)$, cercanamente seguidos de participación en eventos científicos $(\mathrm{N}=322)$ y otros productos varios $(\mathrm{N}=132)$. La presencia en los medios de comunicación también fue importante, con 137 reportajes y entrevistas.

Los artículos en revistas indexadas fueron principalmente en revistas de América Latina ( $\mathrm{N}=147)$ seguido de EEUU ( $\mathrm{N}=90)$, Europa $(\mathrm{N}=79)$ y otras regiones $(\mathrm{N}=46)$. También se reportan 45 artículos en revistas no indexadas.

Las otras publicaciones también fueron numerosas: 89 folletos, 74 capítulos de libro, 60 folletos y volantes, 23 libros y 23 manuales.

En el área docente, hubo 213 cursos beneficiados con el proyecto, produciéndose 242 trabajos finales de graduación, 201 tesis de grado y 145 tesis de postgrado.

A nivel comercial, se lograron 52 productos, 30 procesos y 5 patentes.

Además, 67 investigadores/as continuaron colaborando entre sí tras acabar el proyecto, lo que implica un alto logro en el objetivo de lograr colaboración científica interinstitucional.

Dificultades: El programa también tuvo aspectos débiles. Después del 2010 los fondos asignados por las universidades comenzaron a decrecer, lo que obligó a dar cada vez menos fondos por proyecto, reduciéndose al final a unos $\$ 70000$ por proyecto. En paralelo con esto, el interés por participar en estos proyectos disminuyó y desde ese año se estancó el número de proyectos recibidos en concurso.

Además, cerca de diez proyectos por año no lograron concluir dentro de calendario, obligando a conceder prórrogas, siendo 2015 el peor año en ese sentido. Temáticamente, las humanidades casi no han logrado presentar proyectos con la calidad necesaria, por lo que la distribución temática ha sido la siguiente: 43 proyectos en salud, 37 en ciencias naturales, 22 en agricultura, 21 en tecnología, 12 en ciencias sociales y únicamente tres en humanidades. La colaboración internacional es mínima y se da fundamentalmente con otros países del continente y con Europa Occidental.

\section{DISCUSIÓN}


Dos requisitos éticos básicos en la distribución de fondos es que se realice equitativamente y que produzca el mayor beneficio. Sin embargo, ambos requisitos pueden llegar a ser incompatibles, pues en algunos casos distribuir fondos por igual a todas las instituciones podría resultar en menos fondos para los equipos que produzcan el mayor beneficio (Emanuel et al., 2004; Ballantyne, 2010). Por lo tanto, la distribución debe buscar un equilibrio, y el FEES parece haberlo hallado, porque es poca la desproporción de beneficio entre universidades. Por ejemplo, la Universidad de Costa Rica, que tiene cuatro veces más estudiantes que el Instituto Tecnológico de Costa Rica (CONARE, 2018) solo benefició a 2,6 veces más investigadores que el Instituto Tecnológico de Costa Rica.

En muchos países se busca incrementar la presencia femenina en los equipos de investigación científica, con proyectos como STEM4girls (http://www.stem4girls.org/); y Monge-Nájera \& Ho (2017a) han advertido sobre la escasa participación de mujeres en países como Nicaragua. Sin embargo, la situación en Costa Rica es más cercana a la paridad, con $43 \%$ de investigadoras y un crecimiento mayor de mujeres $(12 \%)$ que de hombres $(8 \%)$ en las carreras científicas (Umaña \& Vargas, 2018). Nuestro análisis calza con estos datos y demuestra que en el FEES, la participación de las mujeres (54\%) supera a la de los hombres, lo que indica un excelente resultado en este campo. Pareciera que una vez más se está invirtiendo el patrón:

"Hasta mediados del siglo 19 - cuando las ciencias se profesionalizaron- eran las mujeres quienes debían estar en el laboratorio, o entre los especímenes geológicos y zoológicos. En aquella época, eran las humanidades las que eran vistas como campos puramente masculinos" (Farago, 2018).

Las preocupaciones válidas en investigación incluyen el que no atiendan los problemas más acuciantes de una sociedad (Ellis \& Levy, 2008) y el de que se archiven en forma de literatura gris que nunca produzca un beneficio real (Jiménez-Medina, 2014). Nuestros hallazgos indican que ese temor no calza con este experimento costarricense, dados la predominancia de proyectos FEES en comunidades poco desarrolladas, y la alta productividad de los proyectos, medida en productos comerciales, actividades comunitarias, artículos científicos, libros, tesis y cursos.

La colaboración científica es valiosa para aumentar la calidad e impacto de la investigación científica, y en América Latina tiende a ser insuficiente a nivel interno de cada país (Russell et al., 2007), y en el caso de Costa Rica, aunque no se ha documentado, la experiencia de los autores es que a fines del siglo 20 había muy poco ambiente para colaboración entre universidades públicas. Precisamente una de las ideas subyacentes en la creación del FEES pudo haber sido la ruptura de las barreras culturales a la colaboración interuniversitaria. En todo caso, el hecho de que decenas de investigadores informen que han continuado colaborando, incluso después de que lo hacían por requisito del FEES, muestra que esa barrera se rompió.

Dos logros que quedan pendientes en esta primera década, son aumentar en lugar de disminuir el financiamiento a los proyectos, y elevar el nivel de los proyectos en humanidades y ciencias sociales, para que sean más competitivos y reciban mayor financiamiento, aunque eso requerirá cambios grandes en ese campo tradicionalmente conservador en cuanto a enfoques y métodos (Monge-Nájera, 2011). 
En conclusión, los proyectos FEES han sido en general exitosos y muy productivos, han beneficiado de manera justa a mujeres y comunidades con mayor necesidad, y representan un ejemplo para otros países pequeños que quieran obtener el máximo provecho de sus limitados fondos de investigación.

\section{AGRADECIMIENTOS}

Agradecemos la colaboración de Cristina Alvarado, Maribel Jiménez, Silvia Sáenz, Sharlín Sánchez, Viviana Salgado, y Maynor Vargas en la toma de datos; y de Elena Vargas y Carolina Seas en la preparación del manuscrito.

\section{Referencias}

Ballantyne, A. J. (2010). How to do research fairly in an unjust world. The American Journal of Bioethics, 10(6), 26-35.

Brenes B., L. (2018). Informe: Investigación y Posgrado en América Central y República Dominicana. San José, Costa Rica: Consejo de Investigación del Sistema Centroamericano de Investigación y Posgrado, Acta 49, artículo 6.

Brenes-Bonilla, L., Bermúdez-Mesén, L. \& Bermúdez-Vargas, K. (2015). SME’s semi-formality rate in Costa Rica: a clusters approach. Faedpyme International Review, 4(7), 47-52. doi:10.15558/fir.v4i7.98

Emanuel, E. J., Wendler, D., Killen, J., \& Grady, C. (2004). What makes clinical research in developing countries ethical? The benchmarks of ethical research. The Journal of infectious diseases, 189(5), 930-937.

Jiménez-Medina, J. A. (2014). Publicar o morir: divulgación de conocimiento en el campo editorial universitario, el caso colombiano. Dialéctica Libertadora, 6, 122-136.

Monge-Nájera, J. (2011). Darwin y su poco impacto en las ciencias sociales costarricenses. Cuadernos de Antropología, 21, 1-6.

Monge-Nájera, J. \& Ho, Y. S. (2012). Costa Rica Publications in the Science Citation Index Expanded: A bibliometric analysis for 1981-2010. Revista de Biología Tropical, 60(4), 1649-1661.

Monge-Nájera, J. \& Ho, Y. S. (2016). Bibliometry of Panama publications in the Science Citation Index Expanded: publication type, language, fields, authors and institutions. Revista de Biología Tropical, 63(4), 1255-1266. doi: 10.15517/rbt.v63i4.21112

Monge-Nájera, J. \& Ho, Y. S. (2017a). Bibliometrics of Nicaraguan publications in the Science Citation Index Expanded. Revista de Biología Tropical, 65(2), 643-655. doi: 10.15517/rbt.v65i2.23985.

Monge-Nájera, J. \& Ho, Y. S. (2017b). Honduras publications in the Science Citation Index Expanded: institutions, fields and authors. Revista de Biología Tropical, 65(2), 657-668. doi: $10.15517 /$ rbt.v65i2.25893

Monge-Nájera, J. \& Ho, Y. S. (2017c). El Salvador publications in the Science Citation Index Expanded: subjects, authorship, collaboration and citation patterns. Revista de Biología Tropical , 65(4), 1428-1436. doi: 10.15517/rbt.v65i4.28397

Monge-Nájera, J. \& Ho, Y. S. (2018). Guatemala articles in the Science Citation Index Expanded: bibliometry of subjects, collaboration, institutions and authors. Revista de Biología Tropical , 66(1), 312-320. doi: 10.15517/rbt.v66i1.29875. 
Russell, J. M., Ainsworth, S., del Río, J. A., J \& Narváez-Berthelemot, N. \& Cortés González, H. D. (2007). Colaboración científica entre países de la región latinoamericana. Revista Española de Documentación Científica, 30(2), 180-198.

Umaña, P. \& Vargas, L. (2018). La participación de las mujeres continúa relegada en las ciencias. Noticias UCR 10 agosto (www.ucr.ac.cr/noticias/2018/08/10/la-participacionde-las-mujerescontinuarelegadaen-las-ciencias.html) 
APÉNDICE DIGITAL 1

Primera década de proyectos de investigación FEES Costa Rica: infogramas de distribución de fondos, productos y tendencias.

Personal beneficiado en los primeros diez años del FEES.

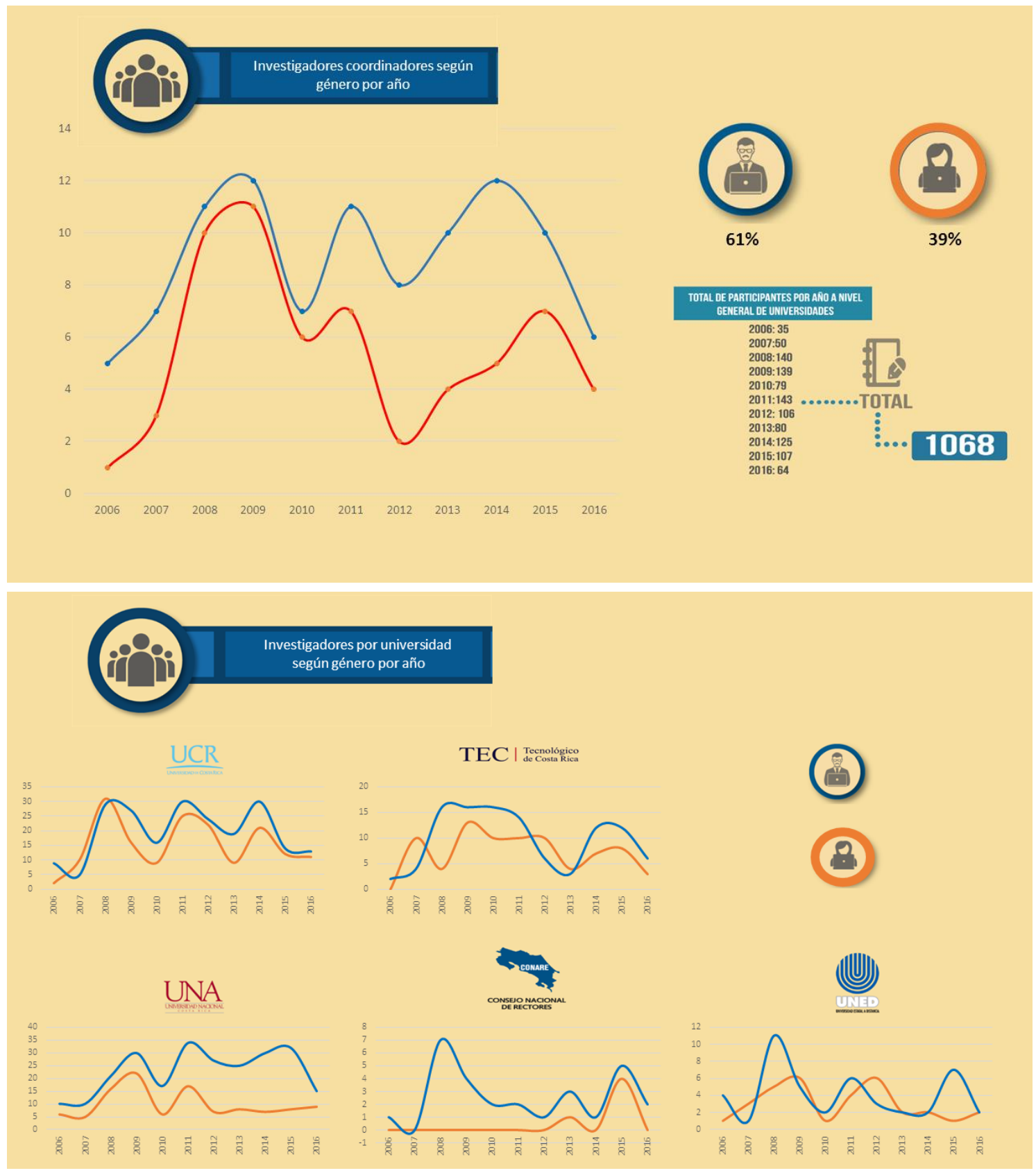


Del 2005 al 2010 hubo un crecimiento fuerte en la cantidad de proyectos, estabilizándose luego de ese periodo:

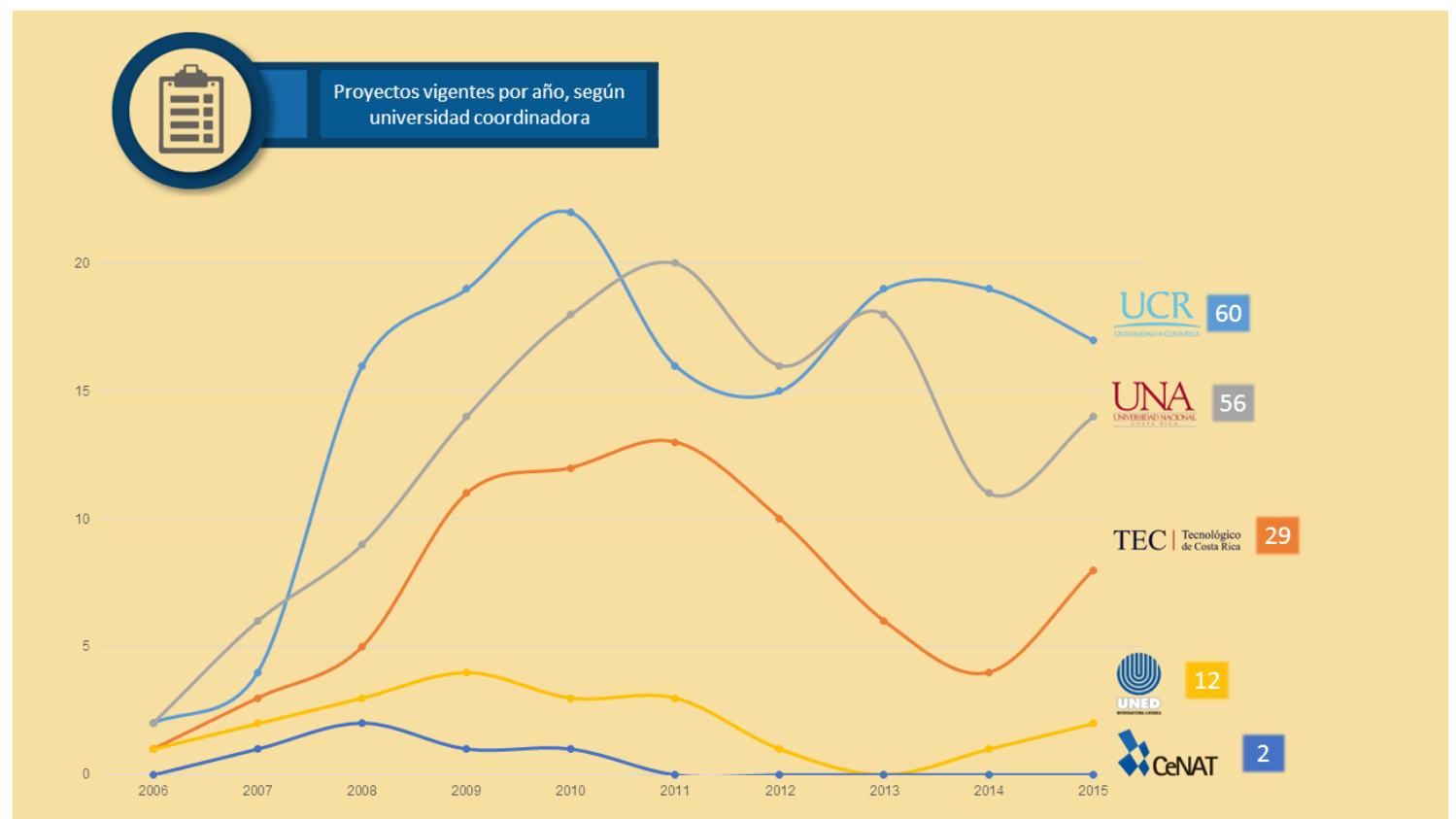

Las solicitudes de prórroga oscilan mucho a lo largo de los años, con un máximo en el 2015:

\section{Solicitudes de prórroga}

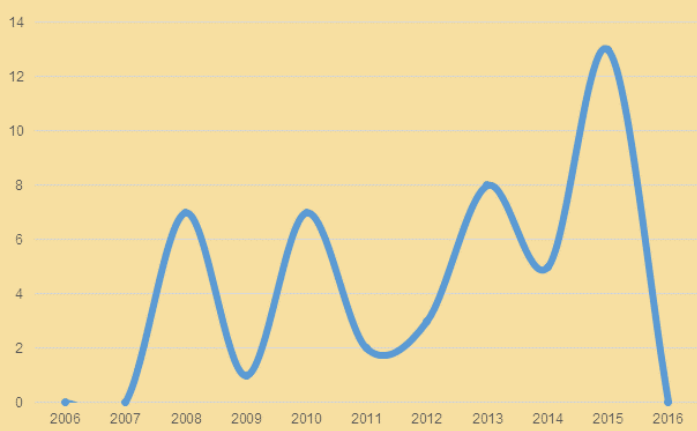


El financiamiento creció marcadamente hasta el 2010 y desde entonces ha venido en descenso:

\section{Financiamiento FS por año}

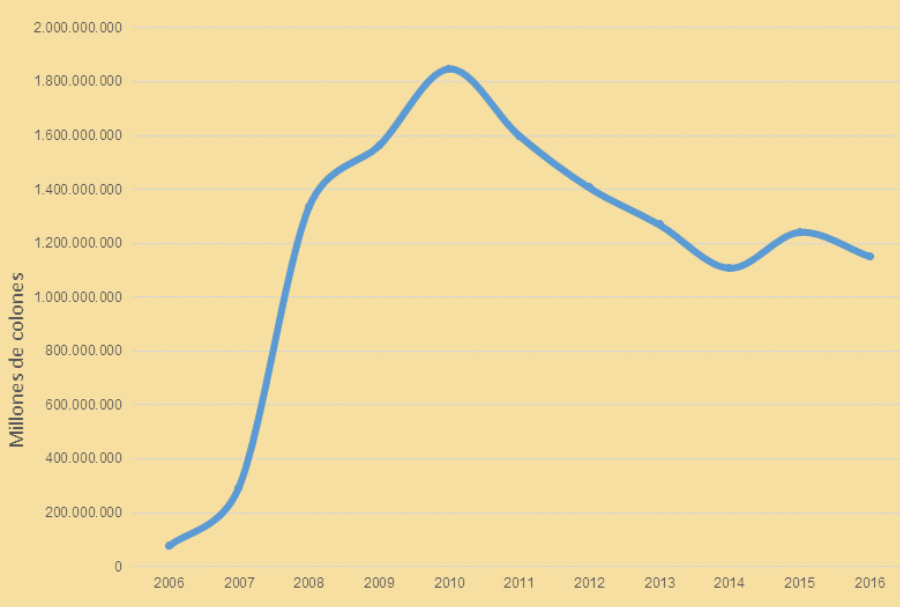


La colaboración con otras instituciones costarricenses ha variado mucho de un año a otro, sin patrón histórico. La colaboración internacional es mínima y se da fundamentalmente con otros países del continente y con Europa Occidental.

\section{Colaboración externa}

\section{Vinculación internacional}
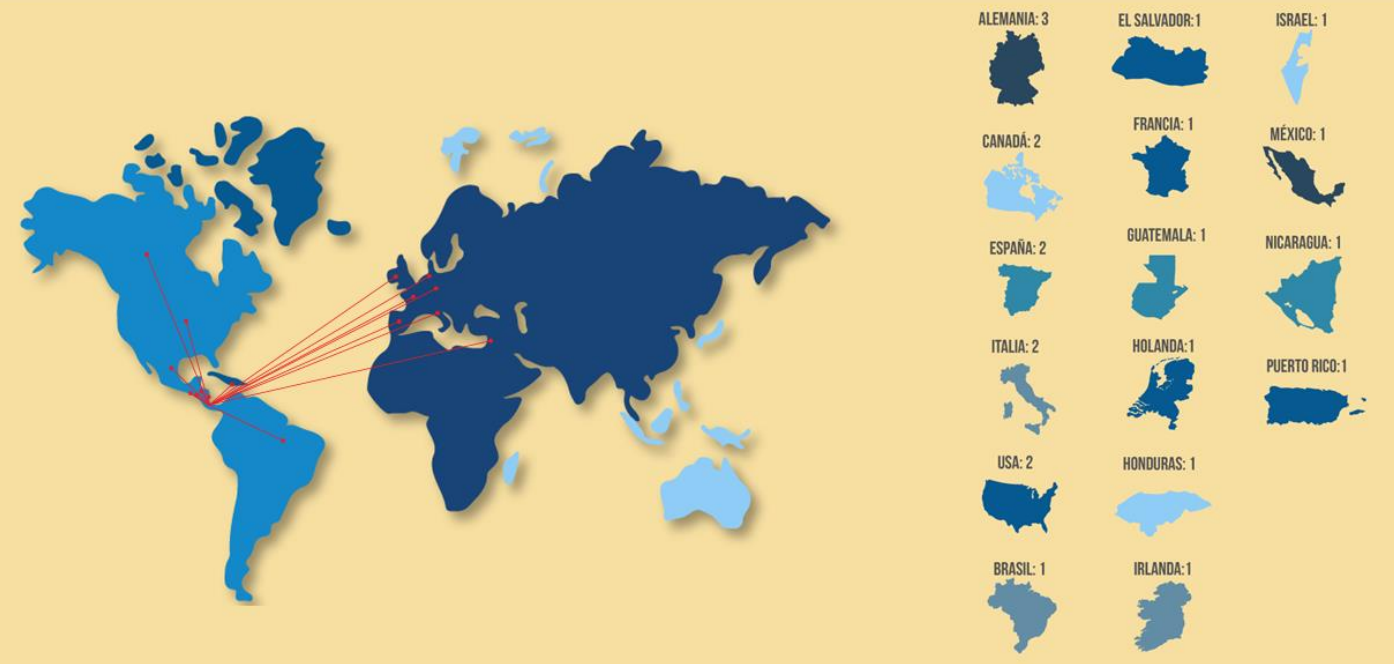
A nivel de provincia, los proyectos han estado centrados administrativamente en la provincia de San José, pero han beneficiado principalmente las zonas costeras (28 proyectos) y las zonas fronterizas (20 proyectos); 32 fueron en la Gran Área Metropolitana.

\section{Provincias}

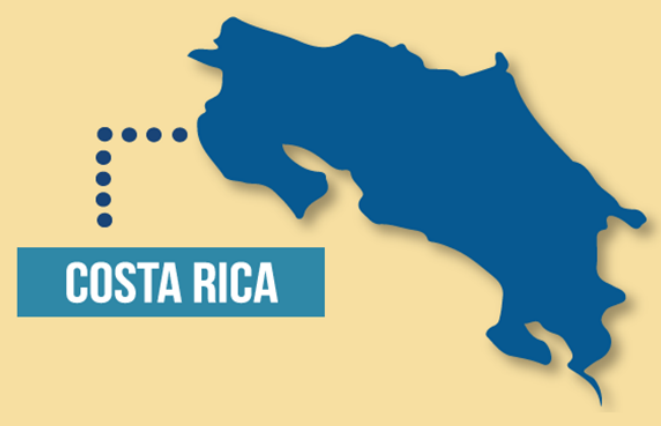

\section{SAN JOSÉ: 42}
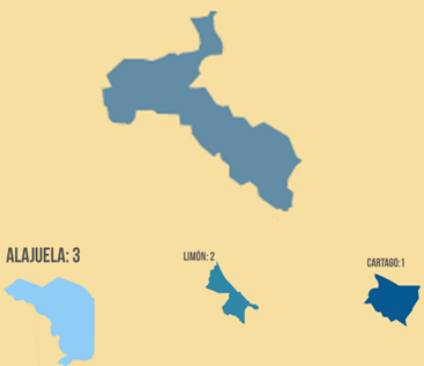

Se han hecho pocos proyectos en humanidades, ciencias sociales, medicina y tecnología. La mayoría han sido en temas agrarios, ciencias exactas y naturales, y con marcado crecimiento en años recientes, estudios multidisciplinarios:
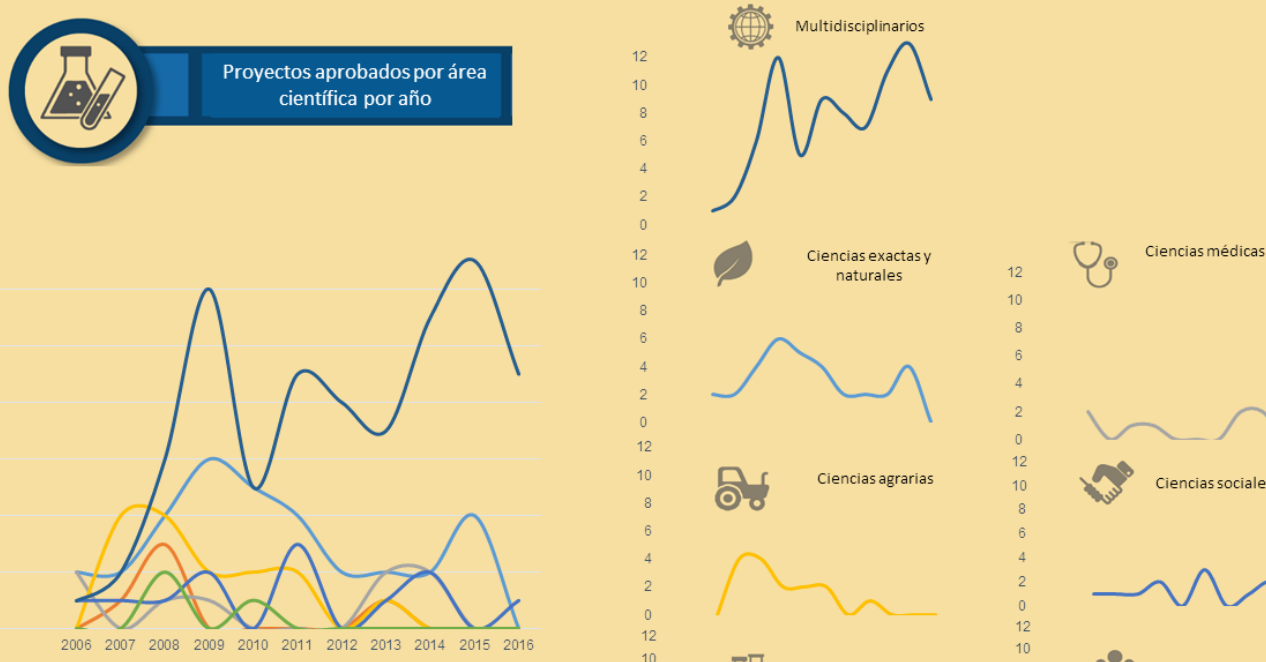

naturales

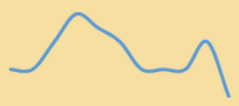

คิ。

Ciencias agrarias
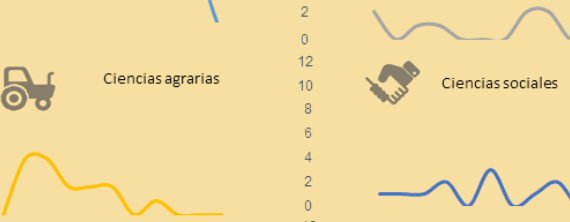

T] Investigacióny tecnologia

\section{Total $=159$}

\section{PROMEDIOS}

Los promedios por proyecto son los siguientes: 


\section{Investigadores por universidad}

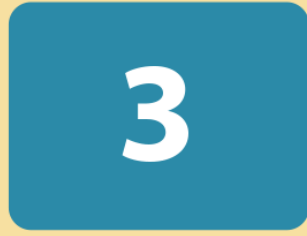

UCR

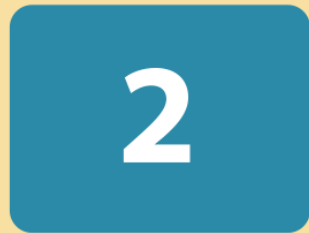

ITCR

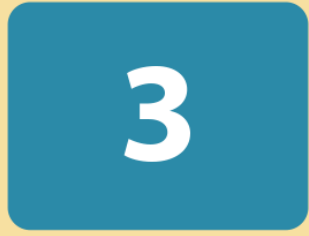

UNA

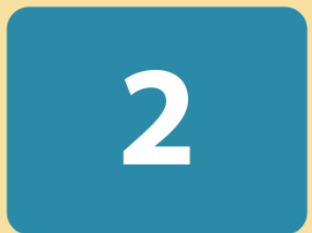

UNED

\section{Investigadores y estudiantes por género}

\section{4}

Investigadoras

\section{4}

Investigadores

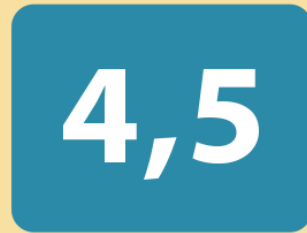

Estudiantes mujeres

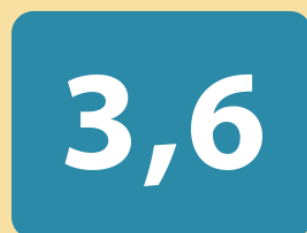

Estudiantes hombres 


\section{Grupos meta o sectores beneficiados}

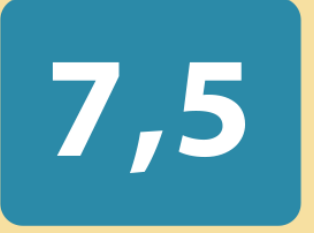

Reuniones con sectores y comunidades

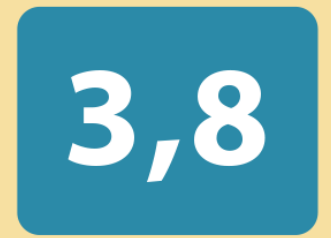

Informes en los medios de comunicación

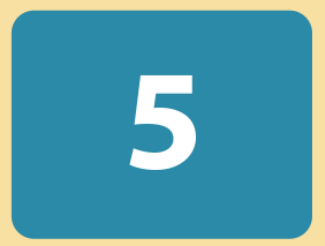

Participación en eventos científicos

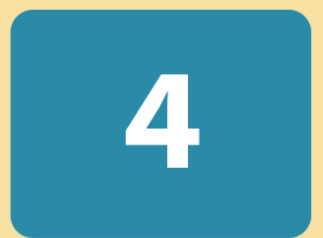

Otros

\section{Publicaciones en revistas indexadas}

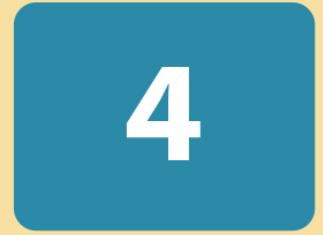

América Latina

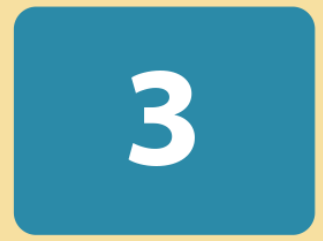

Europa

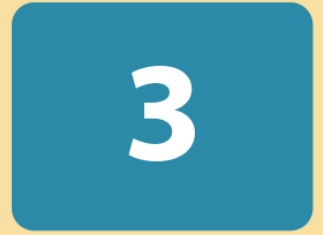

EE.UU.

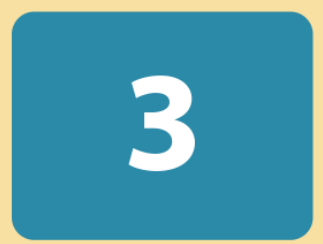

Otras regiones 


\section{Publicaciones por proyecto}

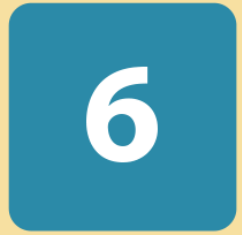

Folletos

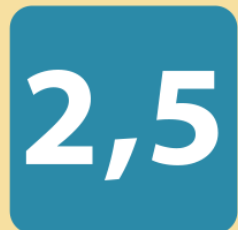

Artículos en revistas no indexadas

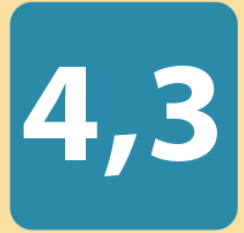

Capítulos de libro

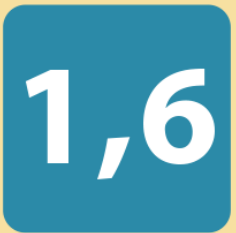

Libros

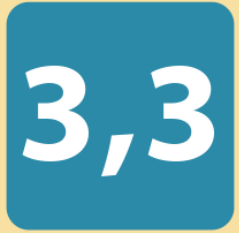

Otras publicaciones (Folletos y volantes)

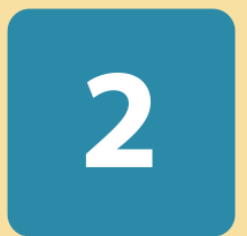

Manuales

\section{Docencia}

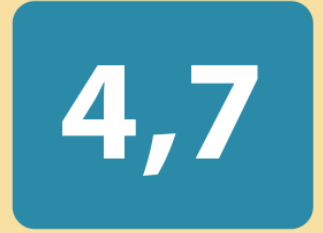

Trabajos finales de graduación

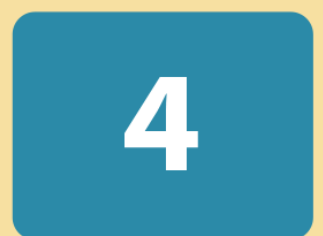

Tesis de grado

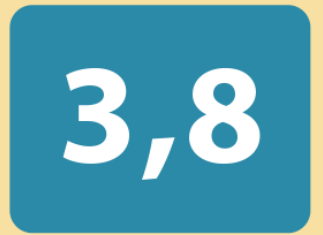

Cursos beneficiados por proyecto

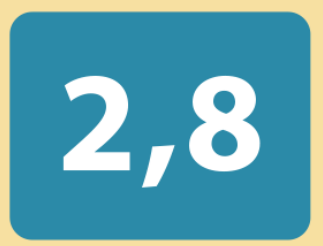

Tesis de posgrado 


\section{Productos de innovación de registro especial}

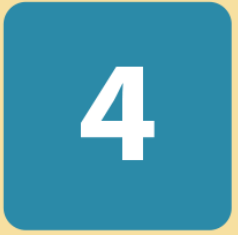

Productos

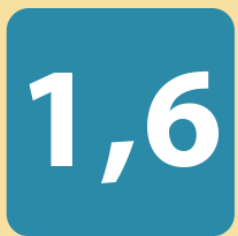

Patentes

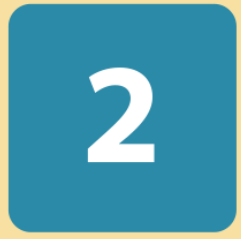

Procesos

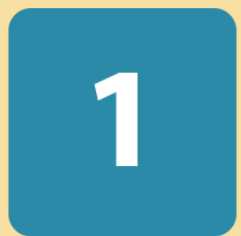

Mercadotecnia

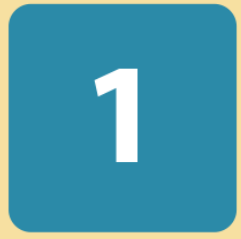

Otros

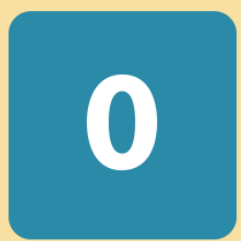

Marcas

\section{Beneficiados por los fondos}

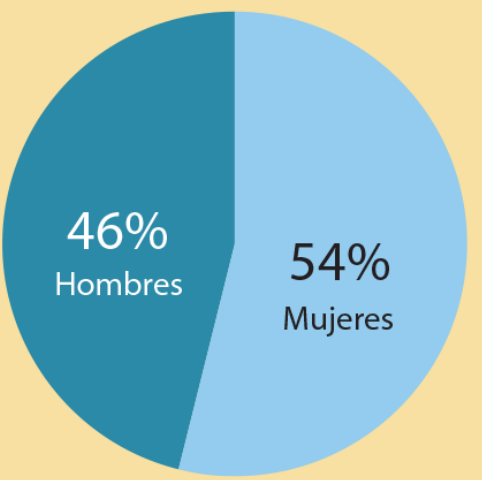

